\title{
The relationship between visual memory and rider expertise in a show-jumping context
}

\author{
Carol Hall $^{\mathrm{a}^{*}}$, Charlotte Liley ${ }^{\mathrm{a}}$, Jack Murphy ${ }^{\mathrm{b}}$, David Crundall ${ }^{\mathrm{c}}$
}

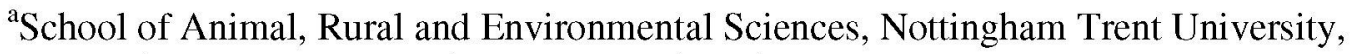
Brackenhurst Campus, Southwell, Nottinghamshire, NG25 0QF, UK

${ }^{\mathrm{b}}$ School of Agriculture, Food Science and Veterinary Medicine, UCD, Dublin, Ireland

${ }^{\mathrm{c}}$ Accident Research Unit, School of Psychology, University of Nottingham, NG7 2RD, UK

* Corresponding Author. Tel.: +44 (0) 1636 817012; fax: +44 (0) 1636815498.

E-mail address: carol.hall@ntu.ac.uk (C. Hall)

\begin{abstract}
Individuals develop visual skills while participating in sport. The aim of this study was to assess the effect of reported riding expertise on the recall of visual information relating to show-jumps. We identified a relevant (F1) and irrelevant (F2) point of focus in 22 photographs of show-jumps. Participants were students $(n=40)$ with varying levels of horse riding ability. The task, having viewed each photograph for four seconds, was to identify F1s or F2s from four alternatives viewed for ten seconds. F1s were recalled significantly more than F2s $(P<0.001)$. Riding expertise did not affect overall recall but only intermediate/advanced riders recalled F1s significantly better than F2s $(P<0.05$ and $P<0.001$ respectively). Recall of F1s but not of F2s was significantly correlated with riding expertise $(P<0.05)$. Training riders in visual attention techniques might improve ridden performance and could reduce the number of accidents associated with lack of experience and/or rider error.
\end{abstract}

Keywords: horse, rider, jumping, vision, training 


\section{Introduction}

Within a sporting context it has been demonstrated that the ability to focus attention on relevant visual information is central to skilful performance. Perceptual and cognitive skills, as well as effective movement patterns, are necessary attributes of elite athletes (Ward and Williams, 2003; Williams et al., 2003). In equestrian sport, where the human participant is often moving at speeds far above those that can be achieved by human propulsion alone, the (human) processing of visual information must occur very rapidly. This rapid response ability is crucial for example, where horse-rider combinations negotiate obstacles such as showjumps at speed. Jumping fences successfully is dependent largely on the ability of both horse and rider to judge not only the fence dimensions but also the distance to the obstacle when cantering or galloping towards it. The ability to adjust the Jump Stride Kinematics (JSK) including approach velocity such that the hind legs of the horse arrive at the appropriate 'take-off' point is critical to maximise the actual jump stride kinematics or bascule over the fence (Laurent et al., 1989). Visual information is particularly important during this phase, when the expertise of both horse and rider will determine the accuracy of the approach, takeoff and bascule.

In show-jumping competitions, riders routinely 'walk the course' to assess the optimal route, various fence combinations and spatial arrangements including distances between obstacles and obstacle dimensions. Experimental trials, using simulations and eye tracking equipment, identified the visual search strategies used by riders when walking a virtual show-jumping course. Differences in the visual search strategies employed by expert and less skilled riders were evident during this simulated task. In general, the more expert riders were much less dependent on the overall course plan than the less skilled riders but they exhibited significantly more fixations on the actual (virtual) fences (Moran et al., 2002). These findings would suggest that show-jumping experience results in differences in visual strategies that are apparent even in simulated situations.

If this is the case during simulated course walking, it is also likely to occur during tasks where individuals might experience other simulated 'approaches' to show-jumping fences. Evidence of expertise-related differences in virtual show jumping tasks would indicate that the latter might be useful during the training of novice riders. Clearly, within equestrian sports, lack of rider expertise and experience will limit overall performance. In fact, given the nature of some equestrian sports, inexperience may increase risk dramatically and present 
serious negative consequences for both horse and rider in some cases. Moreover, analysis of horse riding accidents suggested that of all equestrian activities, incidents associated with jumping was most likely to result in a spinal injury (Silver, 2002).

In a study of factors that affect the severity of horse-related injuries, falling from the horse was found to be the most common cause of injury and most falls (66\%) involved nonprofessional horse riders and handlers (Abu-Zidan and Rao, 2003). Novice riders participating in jumping activities are therefore one of the most 'at-risk' groups and any appropriate training that could increase expertise and minimize risk would be beneficial. Perceptual training programmes based on video simulation, instruction and feedback have been shown to be effective in improving anticipatory skills and in reducing the time taken to make performance related decisions in other sports such as field hockey (Williams et al., 2003). If similar outcomes were possible by training riders (while off the horse) based upon the perceptual skills used by expert riders, then this would improve both safety and performance within equestrian sport. In order to incorporate 'perceptual training' into an equitation coaching schedule, the visual strategies of expert riders must be identified and interpreted.

In one previous study, Laurent et al. (1989) used a video-oculographic gaze recorder to monitor the head orientation and gaze direction of expert riders as they approached an experimental fence. Those authors concluded that when approaching the obstacle, expert riders consistently direct their gaze towards the upper part of the fence, regardless of its configuration. Laurent et al., (1989) have suggested that this is the optimal gaze strategy, which allows riders to assess the time and distance to take-off correctly and that it is based on the retinal expansion rate of the obstacle. The aim of this initial study was to investigate whether previous riding experience and reported expertise would affect recall of visual information relating to pictures of show-jumping fences. If similar features of rider visual search strategies identified in the field (Laurent et al., 1989) were also apparent in such simulated 'laboratory' situations, it would also suggest that simulated visual training could be an effective addition to training riders for show-jumping. 


\section{Materials and Methods}

i) Riding experience and reported expertise of participants

Participants (University students; $n=73$ ) with varying levels of horse riding experience completed a questionnaire. The questionnaire comprised three sections:

In Section 1 participants recorded their gender; age (16-20, 21-25, 26-30 or 30+ years), riding experience (in years) and whether they had passed any riding exams (for example, Pony Club tests or British Horse Society exams). The number of years spent riding was used as a measure of experience in the subsequent analyses.

Section 2 comprised 22 questions relating to confidence and perceived skill in various riding situations, both on the flat (questions 1-10) and when jumping (questions 11-22). The participants rated their answers on a Likert psychometric response scale of 1-5 where the scale indicated their predicted ability to complete specified ridden tasks - for example, ensuring that the horse cantered on the correct lead. The scores ( $1=$ never; $2=$ rarely; $3=$ sometimes; $4=$ quite often; $5=$ always) related to the frequency/likelihood that the subjects could complete each task successfully. The least possible total score (where subjects had no experience of horse riding and scored 1 for each of the 22 questions) was 22 and the greatest was 110 (scored 5 for all 22 questions). The level of expertise was determined for each participant on the basis of their overall scores. They were then assigned to one of four groups accordingly. See Table 1 for the range of scores and associated participant grouping. The study design required equal numbers of subjects in all four groups for the assessment of visual memory. Once the overall scores had been calculated, ten participants from each score range were selected at random. All 73 participants took part in the subsequent visual memory test but only the scores for the 40 that had been selected were used in the final analysis.

Table 1: The four levels of rider expertise as designated by the range of scores gained in Section 2 of the questionnaire.

\begin{tabular}{lll}
\hline Group & Rider expertise & Range of scores \\
\hline E0 & Non-rider & $22-44$ \\
E1 & Novice rider & $45-66$ \\
E2 & Intermediate rider & $67-88$ \\
E3 & Advanced rider & $89-110$ \\
\hline
\end{tabular}


Scores for questions 1-10 (relating to general riding on the flat) and scores for questions 1122 (relating to jumping) were also calculated separately to investigate potential correlations with performance in the subsequent test of visual memory.

Section 3 contained 3 questions that related to the type of horse riding that the participant was mostly involved in (for example, hacking, flat work or jumping); whether they rode competitively and at what level (local/national, novice/advanced).

\section{ii) Assessment of visual memory in a simulated show-jumping context}

In order to assess visual recall in this study, images of fences as they would appear to a rider approaching them on horseback were required. An experimenter (an experienced rider) photographed 22 different show-jumps while mounted on a horse from a position in front of the jump. The height of the jumps varied from a pole on the ground to $110 \mathrm{~cm}$ (mean height $61.82 \mathrm{~cm}$; median height $65 \mathrm{~cm}$ ). The digital photographs were printed in colour on A4 sheets. An elite competitive rider viewed these and identified two points of focus (F1 and F2) for each photograph. F1 was the relevant point of focus when riding towards the fence while F2 was an irrelevant alternative. F1 sections were taken from the central point of the fence; F2 sections could have come from anywhere within the scene. We constructed a multiple-choice slide for each of the 22 photographs, which displayed four comparable sections, one of which was from the original photograph (F1s from 11 photographs and F2s from the other 11 photographs).

The participants had a visual presentation of the 22 photographs, followed by the associated multiple-choice slide after each photograph, from which the subject attempted to select the section that was part of the previous photograph. We labelled the sections $\mathrm{A}, \mathrm{B}, \mathrm{C}$ and $\mathrm{D}$ and participants recorded their answers on prepared sheets which listed the photographs 1-22 and the alternatives A, B, C or D. The presentation was on Microsoft Power Point XP and the slides were set to change automatically throughout the presentation. The presentation of photographs followed by the multiple-choice slide (either F1 or F2) occurred in a random order as either a slide followed by a F1 multiple-choice slide or a slide followed by a F2 multiple-choice slide. Figure 1 shows an example of a photograph of a show jump as used in the presentation (Figure 1a) and the associated multiple choice slide, in this case F1 (Figure 
1b). Each photograph was displayed for four seconds and then the four photo segments for the forced-choice memory test were presented for ten seconds.

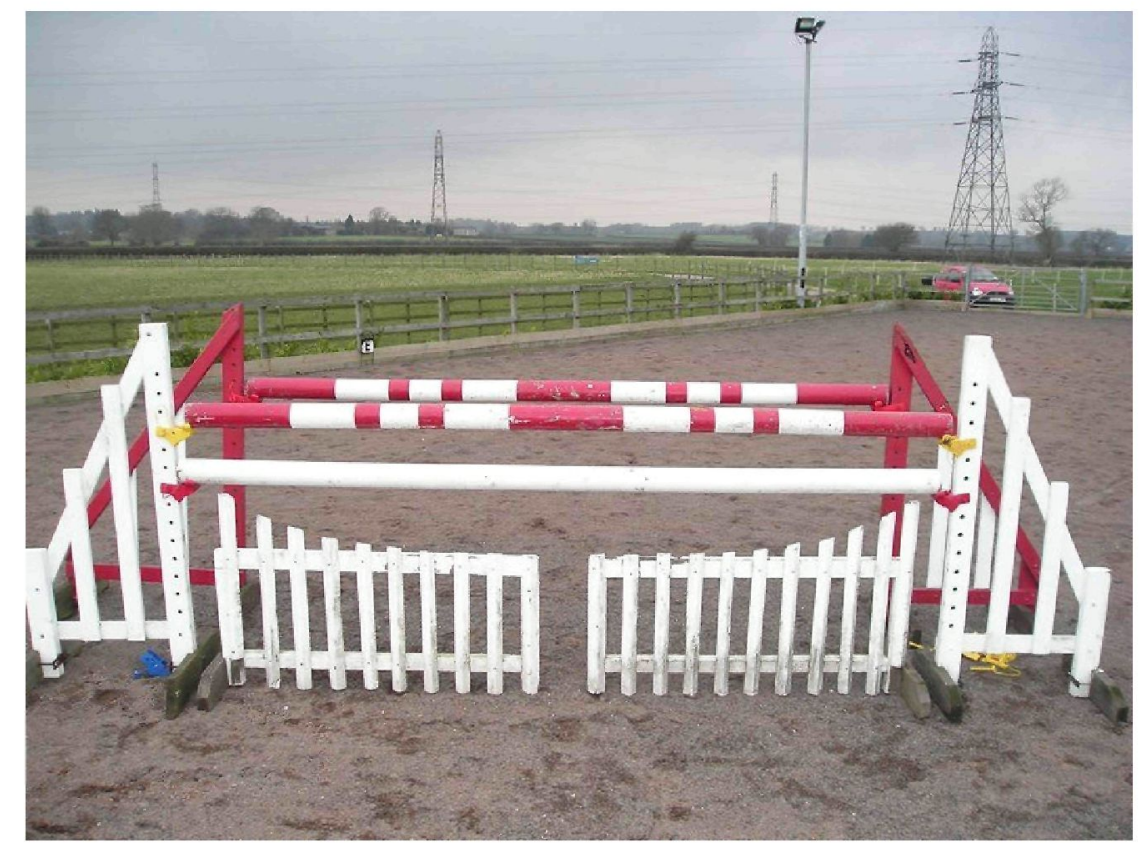

Figure Ia: Photograph of a show-jump as shown in the presentation.

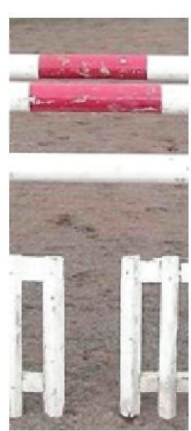

A
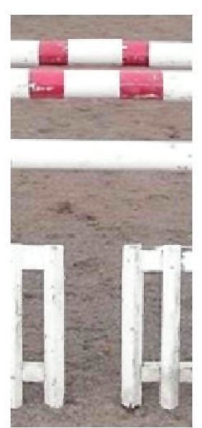

B

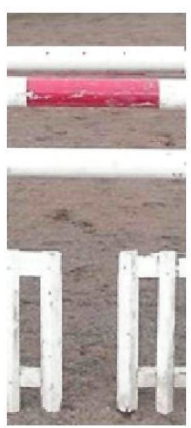

C



D

Figure 1b: F1 multiple-choice slide shown after photograph in Figure 1a. This contains a section from the photograph taken from the relevant point of focus when riding towards the jump (correct choice), as well as three sections not from the original photograph, but taken from the same areas of other similar photographs (incorrect choices). 
We divided the participants ( $n=73$ ) into three different groups $(n \cong 24)$ of mixed riding ability and tested each group for visual recall separately in a classroom setting. The participants saw the presentation on a screen $2.40 \mathrm{~m} \times 1.86 \mathrm{~m}$ from a distance of approximately $4-10 \mathrm{~m}$ and received the following instructions:

"You are about to view a series of photographs of show-jumping fences. Imagine that you are approaching the fence on a horse in order to jump it. You will see a slide (photograph) of the fence for four seconds followed by a slide containing four smaller photos labelled A, B, C and D, one of which originated from the previous photograph. You will see this display for ten seconds and your task is to identify which of the four options originated from the previous photograph. You should record your answers by circling A, B, C or D on the sheets provided."

An example slide followed by a multiple-choice slide was shown to the participants before the test slides. On completion of the task the total number of correct answers was calculated for each of the participants. Correct answers for F1's and F2's were then calculated separately.

iii) Assessment of the relationship between visual memory and rider expertiselexperience

The level of expertise was determined from the responses to Section 2 of the questionnaire, as explained in Section (i) above. Performance in the visual recall task was calculated as total correct responses and whether these correct responses related to F1 or F2 conditions. The data were analysed using SPSS 12 for Windows and a Kolmogorov-Smirnov test for normality identified no significant variation from normal distribution. We used a two-way mixed analysis of variance (ANOVA) to assess the main effects of expertise (E0, E1, E2, E3) and whether the information was relevant to jumping the jump successfully or not (F1, F2), on visual recall. Paired samples $t$ tests were then used to compare recall of relevant/irrelevant information $(\mathrm{F} 1 / \mathrm{F} 2)$ by each expertise group.

We categorised rider characteristics from the responses in Sections 1 and 2 of the questionnaire. The effects of age group, qualification, whether the participant rode competitively or not and whether they regarded jumping as one of their main riding activities or not, were also assessed in relation to visual recall of F1 and F2 information (two-way mixed ANOVA). As $92.5 \%$ of the participants were female, the effect of gender on visual recall was not tested. 
We used Pearson's $r$ to test the correlation between experience (number of years riding) and recall of relevant $(\mathrm{F} 1)$, irrelevant $(\mathrm{F} 2)$ and all $(\mathrm{F} 1+\mathrm{F} 2)$ visual information. We also used Pearson's $r$ to test the correlation between reported expertise and recall of visual information $(\mathrm{F} 1, \mathrm{~F} 2, \mathrm{~F} 1+\mathrm{F} 2)$. Individual scores for the whole of section 2 of the questionnaire and also those from questions 1-10 (general riding and flatwork) and questions 11-22 (jumping) were used in these calculations. We hypothesized that higher levels of experience/expertise would result in improved recall (at least of relevant, F1 information), and consequently 1-tailed tests were used in these analyses.

\section{Results}

In the test of visual recall the mean number of correct responses to the multiple-choice questions, irrespective of the riding experience or reported expertise of the participant, or the relevance of the options, was $11.4( \pm 3.27)$ out of 22 . The mean number of correct responses out of 11 was $6.58( \pm 2.09)$ for the F1 multiple-choice questions and $4.83( \pm 1.84)$ for the F2 multiple-choice questions. Overall, significantly more F1 questions were answered correctly than $\mathrm{F} 2$ questions $\left(F_{(1,36)}=30.668, P<0.001\right)$.

The level of rider expertise did not affect overall performance in the visual recall task ( $\mathrm{F} 1$ and F2 combined correct scores). However, there was a significant interaction between expertise group and whether the information to be recalled was relevant to jumping the fence (F1 or F2) $\left(F_{(3,36)}=3.48, P=0.026\right)$. The effect of reported expertise on the recall of relevant $(\mathrm{F} 1)$ and irrelevant (F2) visual information is shown in Figure 2. In groups E0 (non-riders) and E1 (novice riders) there was no significant difference in the number of correct F1 and F2 answers. However, in group E2 (intermediate riders) significantly more correct responses were recorded for $\mathrm{F} 1$ as opposed to $\mathrm{F} 2$ questions $\left(t_{(9)}=2.492, P=0.034\right)$. The difference between the number of correct responses to F1 and F2 questions was even greater in group E3, the most expert riders $\left(t_{(9)}=5.82, P<0.001\right)$.

The were no significant effects related to age group, qualification, whether the participant rode competitively or not and whether show-jumping was one of their main riding activities, on the total number of correct answers. Equally, there was no significant interaction between any of these factors and whether the information to be recalled was relevant to jumping the jump or not (F1 or F2). 
When the reported expertise (Section 2: questions 1-22) of individual participants was compared with their performance on the visual recall test, a positive correlation between overall expertise and the number of correct answers to F1 questions was found ( $r=0.27, n=40$, $P=0.045$ ). When the reported expertise in relation to jumping only (Section 2: questions 1122) was compared with the number of correct answers to F1 questions, the resulting correlation was slightly stronger $(r=0.29, n=40, P=0.035)$. No correlation was found between reported general riding expertise (Section 2: questions 1-10) or experience (Section 1: number of years spent riding) and the number of correct answers to F1 questions. No correlation was found between the reported expertise or experience of participants and their overall performance on the visual recall test (combined correct scores $\mathrm{F} 1+\mathrm{F} 2$ ) or performance with the F2 questions.

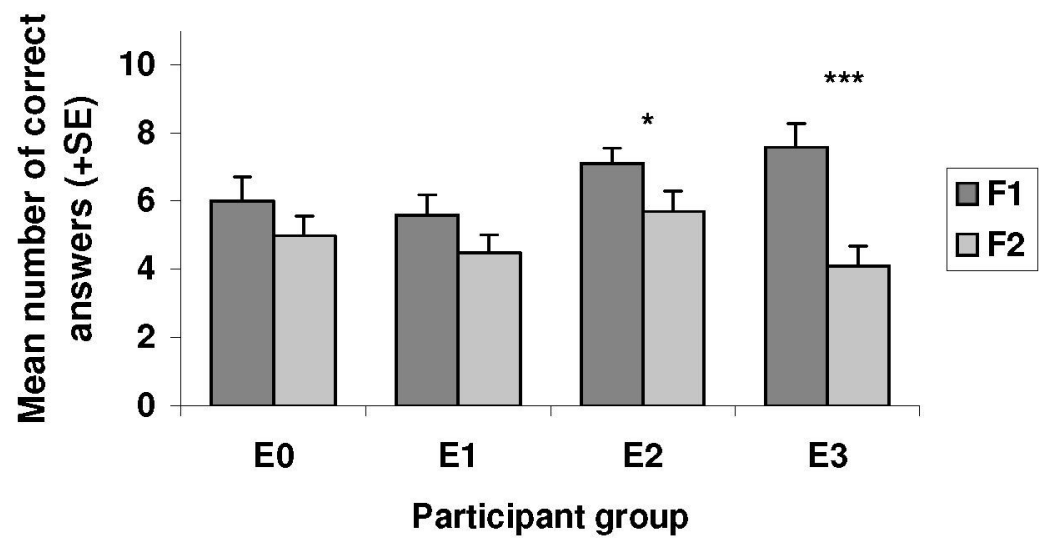

Figure 2: The effect of reported expertise on the recall of relevant $(F 1)$ and irrelevant $(F 2)$ visual information. ${ }^{*}$ denotes significance at 0.05 level; ${ }^{* * *}$ denotes significance at 0.001 level.

\section{Discussion}

The findings from the current study indicated that factors relating to the participants' actual performance in the sport of show jumping influenced the recall of visual information from the photographs of the show-jumping fences. While approaching a fence when show jumping, expert riders direct their gaze to the highest central point of the fence (Laurent et al., 1989). When constructing the multiple-choice slides the images were taken from this area of the photographs of show jumps for the F1 (relevant visual information) condition. The 
participants in this study correctly identified these sections (F1) significantly more frequently than the irrelevant alternatives (F2), which were images taken from other areas within the scene. However, this difference was only significant for those participants who had actual experience in horse riding, and more particularly the individuals with previous experience of jumping fences. In all cases, we found a significant positive correlation between the reported expertise of the subjects and the recall of the relevant information from the multiple-choice slides. The results support the findings of Laurent et al. (1989) and provide further evidence that visual skills (developed from experience in the equestrian sport of show jumping) will also affect performance in simulated tasks.

The visual strategy used by experienced riders differs from that of novice riders when these riders are planning how to approach and jump obstacles in equestrian sport. Experienced riders appear to assimilate more visual information relating to the jump than do more novice riders (Moran et al., 2002), as was implied by the results of the present study. It is possible that the more expert/experienced riders directed more visual attention to the part of the jump that relates to successful negotiation of the obstacle when mounted. It is equally possible that their familiarity with such obstacles resulted in them being able to recall the visual features of the jumps more readily than features taken from other areas of the scene.

A few of the photographs depicted relatively large jumps (one obstacle was $110 \mathrm{~cm}$ high) that could cause anxiety in those riders who had minimal experience in the sport. In some instances location-specific anxiety has been shown to draw attention towards the location responsible, for example, weapon-focus (Loftus et al., 1987). If in this case the pictures of the larger jumps had provoked an anxiety response in the more novice riders, their attention should have been drawn to the jump and as a result their memory for jump features would have been improved. However, this was not the case and it is unlikely that anxiety had any effect on performance in this simulated task.

Whatever the reason for their improved performance in relation to the relevance of the section viewed, experience within the sport appears to have impacted on the visual search strategies used by the participants in this simulation. This phenomenon is a function of the rider's ability to utilise visual information and place the horse in an optimum position to jump fences successfully. Clearly, the ability of expert riders to both judge the time and distance to take-off, and to control the approach and take-off point of the horse, will be greater than that of novice riders. Maintaining a steady and consistent point of focus at the highest central part 
of the jump facilitates the judgement of pace, time and distance to take-off on the basis of visual information (retinal expansion rate). The associated steadiness of the rider's head will also minimise the extent to which the horse is unbalanced by weight shifts of the rider when approaching the jump.

The show-jumping fences displayed in the presentation in this study were generally located near the centre of the photograph. As the relevant (F1) sections were taken from the centre of the jump, this would tend to be the first point viewed by the participants, regardless of showjumping experience, as indicated by the overall better recall of F1 sections. However, the more expert riders were the only ones to have significantly better recall of $\mathrm{F} 1$ as compared with F2 sections, indicating that expertise affects recall performance. We suggest that it may be possible to train novice riders to focus on the central highest point of the fence by focussing on the image in photographic displays. Moreover, by using more animated simulations, novice riders could learn to maintain this point of visual focus even if the image was appearing to move and/or increasing or decreasing in size. This simple approach might prove beneficial as a means of creating pseudo 'riding' toward a fence in training for jumping. In essence, participants could learn to direct their gaze consistently towards the central highest point of any fence by projecting 'moving' or transitional images of the fences on a screen. We suggest that as has been shown in other sports (Williams, 2002; Williams et al., 2003), simple perceptual training of this nature could improve both ridden performance and reduce the number of accidents that result from rider error.

Stachurska et al. (2002) have shown that certain fence types are problematic for show jumping horses and it appears that some 'visual' features of these fences specifically affect performance. It appears that the shape/configuration and colour of the fences including individual fence position within a course of fences determined how well the horse-rider combinations jumped each fence. However, it also appears that much more detailed investigations are necessary to determine if these factors relate specifically to rider error, horse error or a combination of both. We suggest, based on our findings here, that simulated jumping situations might prove useful to assess rider performance in relation to different fence types. If successful, this approach could form the basis for further perceptual training programmes to improve rider performance in show jumping and other equestrian sports. 


\section{Conclusion}

Participants were more successful in recalling relevant information (required to jump fences optimally) from images of actual show-jumping fences than less relevant image details. The visual recall ability of riders depends upon actual rider experience of the sport when tested in a simulated show-jumping context. More expert riders recalled significantly more relevant information in relation to the images of the fences than did novice riders. The use of visual training programmes has benefited participants in other sports and similar outcomes appear possible in equestrian sports with bespoke visual training programmes. Training of this nature is likely to result in improved ridden (and jumping) performance and a reduction in the number of riding accidents that result from rider error.

\section{References}

Abu-Zidan, F.M., Rao, S., 2003. Factors affecting the severity of horse-related injuries. Injury 34, 897-900.

Laurent, M., Dinh Phung, R., Ripoll, H., 1989. What visual information is used by riders in jumping? Human Movement Science 8, 481-501.

Loftus, E.F., Loftus, G.R., Messo, J., 1987. Some facts about “weapons focus". Law and Human Behavior 11, 55-62.

Moran, A., Byrne, A., McGlade, N., 2002. The effects of anxiety and strategic planning on visual search behaviour. Journal of Sports Sciences 20, 225-236.

Silver, J.R., 2002. Spinal injuries resulting from horse riding accidents. Spinal cord 40, 264271.

Stachurska, A., Pieta, M., Nesteruk, E., 2002. Which obstacles are most problematic for jumping horses? Applied Animal Behaviour Science 77, 197-207.

Ward, P., Williams, A.M., 2003. Perceptual and cognitive skill development in soccer: the multidimensional nature of expert performance. Journal of Sport and Exercise Psychology 25, 93-111.

Williams, A.M., 2002. Perceptual and cognitive expertise in sport. The Psychologist 15, 416417.

Williams, A.M., Ward, P., Chapman, C., 2003. Training perceptual skill in field hockey: is there transfer from the laboratory to the field? Research Quarterly for Exercise and Sport 74, 98-103. 